\title{
VORWORT DES VERFASSERS ZUR DEUTSCHEN AUSGABE
}

Ende der vierziger Jahre wurden die ersten Untersuchungen über lineare Optimierungsprobleme durchgeführt. Zuvor hatte man in der Mathematik nur solche Extremalaufgaben behandelt, bei denen Funktionale mit gewissen Differenzierbarkeitseigenschaften betrachtet wurden, die auf offenen Mengen definiert sind. Die lineare Optimierung leitete ein neues Kapitel in der mathematischen Optimierungstheorie ein, weil damit Grundlagen zur Berücksichtigung sogenannter Randeffekte geschaffen wurden. Ein wesentlicher Teil des vorliegenden Buches gibt eine detaillierte Darstellung solcher Grundlagen für allgemeine Probleme der linearen und nichtlinearen Optimierung in unendlichdimensionalen Räumen.

Obwohl eine allgemeine Optimierungstheorie fehlte, traten in der Mathematik auch schon früher spezielle Optimierungsprobleme mit Ungleichungen als Nebenbedingungen auf, wobei die Untersuchungen jedesmal die Ausarbeitung spezieller Methoden und Verfahren erforderten. Besonders viele derartige Probleme kamen in der konstruktiven Funktionentheorie vor. Aus diesem Grunde wurden von mir eine Reihe geeigneter Optimierungsprobleme dieses klassischen Gebietes der Mathematik ausgewählt, um hieran die Bedeutung der im Buch gemachten Aussagen aufzuzeigen. Die im Buch entwickelten Methoden gestatten es, bekannte Resultate zu bestätigen und weiterzuentwickeln.

Es freut mich sehr, daß dieses Buch in der Deutschen Demokratischen Republik erscheint, wo eine große Anzahl von Mathematikern erfolgreich Theorie und Lösungsverfahren für Optimierungsprobleme weiterentwickelt. Meine tiefe Dankbarkeit gilt den Kollegen, die die nicht leichte Arbeit auf sich genommen haben, das Buch in die deutsche Sprache zu übersetzen.

16. 07. 1974

E. G. Golsten 
